\title{
PRELIMINARY STUDY TOWARDS A NOVEL EXPERIMENTAL MODEL TO STUDY LOCALIZED CUTANEOUS LEISHMANIASIS CAUSED BY Leishmania (Leishmania) mexicana
}

Erika Ivett SOSA-BIBIANO, Nicole Raymonde VAN WYNSBERGHE, Silvia Beatriz CANTO-LARA \& Fernando Jose ANDRADE-NARVAEZ

\begin{abstract}
SUMMARY
There is not an experimental model of localized cutaneous leishmaniasis (LCL) caused by Leishmania (Leishmania) mexicana. The aim of the present study was to characterize the clinical and histological features of Peromyscus yucatanicus experimentally infected with $L$. (L.) mexicana. A total of 54 P. yucatanicus (groups of 18 ) were inoculated with $1 \times 10^{6}$ promastigotes of $L$. (L.) mexicana in the base of the tail. They were euthanized at three and six months post experimental infection. The control group was inoculated with RPMI-1640. The predominant clinical sign observed was a single ulcerated lesion in $27.77 \%(5 / 18)$ and in $11.11 \%(2 / 18) P$. yucatanicus at three and six months respectively. The histological pattern described as chronic granulomatous inflammation with or without necrosis was found in 7/7 (100\%) biopsies of euthanized P. yucatanicus at three $(\mathrm{n}=5)$ and six $(\mathrm{n}=2)$ months, respectively. These results resembled clinical and histological features caused by $L$. ( $L$. ) mexicana in humans, and support the possibility to employ P. yucatanicus as a novel experimental model to study LCL caused by this parasite.
\end{abstract}

KEYWORDS: Peromyscus yucatanicus; Leishmania mexicana; Symptoms; Histology.

\section{INTRODUCTION}

Localized cutaneous leishmaniasis (LCL) known as "chiclero's ulcer" (collector of natural chewing gum) in southern Mexico, was described by Seidelin in $1912^{31}$. Since then, the forest region of the Yucatan peninsula has been recognized as an endemic focus of $\mathrm{LCL}^{3,6,32}$. Leishmania (Leishmania) mexicana Biagi, 1953, emend. Garnham, 1962, is the main agent causing CL in this focus; however, L. (Viannia) braziliensis has also been isolated in a few cases ${ }^{9,10}$.

The clinical features of "chiclero's ulcer" have been studied and it is characterized predominantly by cartilage involvement of the pinna of the $\operatorname{ear}^{7,16,39}$. The most frequently observed presentation is a single, ulcerated, rounded small lesion, with elevated borders and necrotic center, located on the ear, with an evolution time of less than three months, and with neither cutaneous metastases nor lymphatic nor mucosal involvement ${ }^{4}$. Criteria for scars resulting from healed lesions of humans with CL caused by Leishmania (Viannia) braziliensis include: no history of trauma, round or oval shape, smooth surface, depressed, and pigment change, hypopigmentation $^{38}$. No differences have been observed in scars from LCL caused by L. (L.) mexicana (unpublished data).

The histopathology of LCL due to Leishmania (Leishmania) mexicana in the Yucatan Peninsula, Mexico, has also been described. Type III and IV of Magalhaes' classification ${ }^{21}$ have been found as the most common patterns in 21/73 (28.7\%) and 32/73 (43.8\%) respectively of our LCL cases caused by L. (L.) mexicana ${ }^{5}$.

The cutaneous leishmaniasis laboratory mouse model using C57BL/6 (resistant) and BALB/c (susceptible) infected with L. major is the most employed system for analyzing the regulation of the immune response against this pathogen only contained by a cell-mediated response ${ }^{19,30}$. However, C57BL/6 mice inoculated with $L$. (L.) mexicana developed a chronic disease ${ }^{2}$. Although the L. major mouse model is considered by many to be the best small animal immunological model for CL, concern about its relevance suggests other models should be developed. In particular, because laboratory mice are usually reduced to homozygosity, are hybrids of three species (Mus musculus musculus, M. m. domesticus,

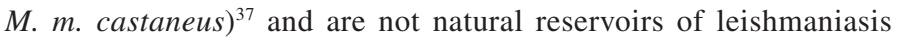
parasites, the use of other, wild-type species that are naturally infected should be considered as alternative models.

In Campeche, three rodents have been identified as primary reservoirs of L. (L.) mexicana: Ototylomys phyllotis Merriam, 1901; Peromyscus yucatanicus J.A. Allen and Chapman, 1897; and Heteromys gaumeri J.A. Allen and Chapman, 1897 10,11,35,36. The two latter species are endemic in the Yucatan Peninsula. O. phyllotis and P. yucatanicus have been adapted to laboratory and a colony for experimental studies has been established at our institution, however the former has been rejected to be employed as an experimental model to study CL caused by $L$. $\left(L\right.$.) mexicana ${ }^{14}$. The

Financial support: Consejo Nacional de Ciencia y Tecnologia (CONACYT), México, ID: 33211-M.

Centro de Investigaciones Regionales Dr. Hideyo Noguchi, Universidad Autonoma de Yucatán, Ave. Itzaes No. 490 x 59, CP 97000, Mérida, Yucatán, México.

Correspondence to: Fernando Jose Andrade-Narvaez. E-mail: anarvaez@uady.mx 


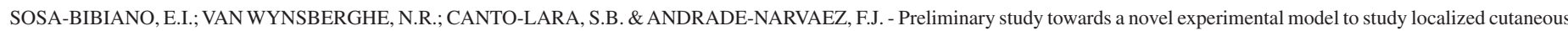
leishmaniasis caused by Leishmania (Leishmania) mexicana. Rev. Inst. Med. Trop. Sao Paulo, 54(3): 165-9, 2012.

use of local mammalian fauna as a model host to study the development of experimental Leishmania spp. infection could give a more approximate whole picture of what probably occurs in natural conditions ${ }^{12}$.

In order to develop a novel experimental model of LCL caused by $L$. (L.) mexicana we sought to characterize clinical and histological features of $P$. yucatanicus experimentally infected with $L$. (L.) mexicana as a fundamental first step for developing an infection model.

\section{MATERIALS AND METHODS}

Animals: Six month old $P$. yucatanicus of both sexes were selected from the colony. The animals were maintained in the animal care facility of the Centro de Investigaciones Regionales, Universidad Autonóma de Yucataán (CIR, UADY). The mice were housed individually in small cages $(19 \times 29 \times 12 \mathrm{~cm})$ lined with wood shavings and fed ad libitum rodent chow (2018S Harlan, Madison, Wisconsin, USA). For enrichment and food balance, once a week the mice were given fresh fruit or vegetables. During the experiment, the animals were kept at $22{ }^{\circ} \mathrm{C}$ with a 12/12h light cycle. Physical enrichment was provided weekly in the form of cardboard tubes for hiding and paper for nesting material. All animals were euthanized with sodium pentobarbital $100 \mathrm{mg} / \mathrm{kg}$, according to the Mexican Law for the use of laboratory animals, and the Guide for the Care and Use of Laboratory Animals ${ }^{13,29}$. The proposal was approved by the bioethics committee of CIR, UADY.

Parasite: Although the dose of Leishmania promastigotes delivered by the sand fly vector to the skin of mammalian host has never been directly determined, using a real time-PCR approach it has been possible to reveal a remarkable range of 10 to 100,000 in the dose of parasites transmitted to mice exposed to a single infected sand fly ${ }^{15}$. More recently, another study determined infection parameters in the sand fly vector that predict transmission of $L$. (L.) major: it was estimated in the range of $10^{3}$ to $10^{6}$ total parasites per $\mathrm{fly}^{34}$. Taking into account these studies we chose to inoculate $1 \times 10^{6}$ promastigotes to increase the possibility of inducing CL clinical signs.

Local strain MPERO/MX/97/Py4, isolated from a $P$. yucatanicus with clinical signs (ulcerated lesion), was identified by isoenzymes and monoclonal antibodies as $L$. (L.) mexicana and was selected for use in this study ${ }^{9,10}$. Its infectivity was restored by a passage in Syrian golden hamsters (Mesocricetus auratus). Promastigotes were grown in Senekjie's medium for seven days at $23{ }^{\circ} \mathrm{C}$. Stationary-phase promastigotes were washed three times in RPMI-1640 medium (Gibco) before being counted and adjusted to a $1 \times 10^{6}$ concentration for inoculation at a final volume of $40 \mu \mathrm{L}$. All animals were inoculated in the base of the tail.

Parasite demonstration: Based on previous studies in which local signs of infection by L. (L.) mexicana were always located at the base of the tail $1^{10,11,35,36}$, smears were made from samples collected at the edge of the lesion (avoiding ulcerated and/or heavily crusted parts) or from the inoculation site in absence of local signs. Moreover, smears were made from the liver and spleen samples. Furthermore, aspirates and cultures from the skin of the base of the tail in each animal were prepared. Liquid culture medium (0.1-0.2 mL RPMI 1640) with antibiotics were inoculated and aspirated repeatedly with an insulin needle ( $1 \mathrm{~mL}, 27 \mathrm{G} \times 13 \mathrm{~mm})$ subcutaneously into base of the tail or at the margin of the lesion when it existed. The aspirates were cultured into tubes containing modified
Senekjie's medium, incubated at $23.5^{\circ} \mathrm{C}$ and examined weekly by light microscope for parasite growth. A culture was considerate positive when at least one promastigote was observed and negative if no parasites were found. If negative, the procedure was repeated until three consecutive aspirates were found with one week intervals.

Experimental infection: A total of $54 P$. yucatanicus in groups of 18 animals were inoculated in the base of the tail and were euthanized at three (group I) and six (group II) months post experimental inoculation. Control group (III) was inoculated with RPMI-1640 and euthanized at three months.

Clinical features: All animals were examined weekly for the following local clinical signs of infection including ulcerated lesion, crust and scar, which are the main documented symptoms in humans with LCL caused by L. (L.) mexicana ${ }^{4}$.

Histological features: Skin biopsies were taken from the edge of the lesion (avoiding ulcerated and/or heavily crusted parts) or from the inoculation site in absence of local signs with a 4-mm disposable punch and $2 \%$ xylocaine as an anesthetic just before euthanizing the animals. The material was fixed in neutral formalin, routinely processed and embedded in paraffin. Sections were stained with hematoxylin and eosin. Histopathological findings were grouped in patterns according to a classification proposed by MAGALHAES et al. ${ }^{21}$. The following five histopathological patterns were defined as having occurred and it has been done in CL patients infected with $L$. (L.) mexicana: type I) exudative-cellular reaction due to infiltration of histiocytes, lymphocytes, and plasma cells, without granuloma; type II) exudative-necrotic reaction, characterized by cellular infiltration, and necrosis and no granulomatous response; type III) exudative and necrotic-granulomatous reaction corresponding to the pattern described as chronic granulomatous inflammation with or without necrosis; type IV) exudative granulomatous reaction (unorganized granuloma) without necrosis characterized by the presence of an unorganized granulomatous reaction; type V) exudative tuberculoid reaction in which a typical tuberculoid granuloma (organized) is formed.

\section{RESULTS}

Clinical features: Local clinical signs were recorded for each one of the experimental groups inoculated with $1 \times 10^{6}$ promastigotes of $L$. (L.) mexicana. None of the P. yucatanicus inoculated with RMPI-1640 developed clinical or histological features of Leishmania infection. Absence of local clinical signs was observed in a total of 29/36 (80.55\%) of the $P$. yucatanicus experimentally inoculated including both groups I and II. At three months, Group I, the most common clinical sign was a single ulcerated lesion observed in $27.77 \%$ (5/18) P. yucatanicus that completed the study, within a range of eight to 10 weeks post experimental infection and persisting without healing during the whole period of survey. In $P$. yucatanicus followed for six months, Group II, only in $2 / 18(11.11 \%)$ ulcerated lesions and crusts were observed, and healing was recorded at week 17 and 18 respectively. Results regarding clinical features are shown in Table 1 and Figure 1. There was no difference in the course of the infection linked to $P$. yucatanicus gender.

Histological features: At three months the histological picture of Leishmania infection was characterized by pattern type III, i.e. exudative 


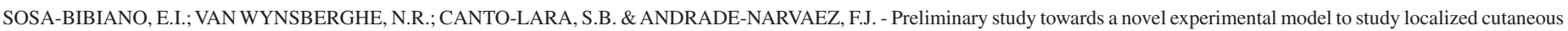
leishmaniasis caused by Leishmania (Leishmania) mexicana. Rev. Inst. Med. Trop. Sao Paulo, 54(3): 165-9, 2012.

Table 1

Local clinical signs developed by $P$. yucatanicus experimentally infected with $1 \times 10^{6}$ promastigotes of Leishmania (Leishmania) mexicana

\begin{tabular}{lcccc}
\hline P. yucatanicus & Group & Ulcer & Crust & Scar \\
\hline Py-9 & 3 months & Weeks 8-12 & Weeks 9-12 & \\
Py-10 & 3 months & Weeks 9-12 & Weeks 9-12 & \\
Py-11 & 3 months & Weeks 10-12 & Weeks 9-12 & \\
Py-12 & 3 months & - & Weeks 9-12 & \\
Py-15 & 3 months & Weeks 8-12 & - & \\
Py-16 & 3 months & Weeks 10-12 & Weeks 10-12 & \\
Py-42 & 6 months & Weeks 8-10 & Weeks 11-17 & Weeks 18-24 \\
Py-43 & 6 months & Weeks 16-17 & Weeks 16-17 & Weeks 17-24 \\
\hline
\end{tabular}
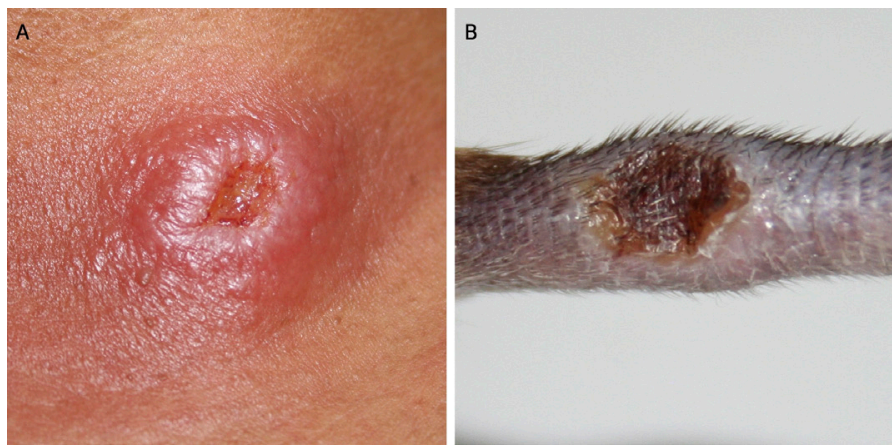

Fig. 1 - A: typical patient localized cutaneous nodular ulcerated lesion caused by $L$. $(L$.) mexicana; B: representative nodular ulcerated lesion from a $P$. yucatanicus experimentally infected with $1 \times 10^{6}$ promastigotes of $L$. (L.) mexicana.

and necrotic-granulomatous reactions corresponding to the pattern described as chronic granulomatous inflammation with or without necrosis were found in 7/7 (100\%) biopsies of euthanized $P$. yucatanicus at three $(\mathrm{n}=5)$ and six $(\mathrm{n}=2)$ months respectively. Parasites were observed in all P. yucatanicus with local signs of infection.

Parasite demonstration: Smears from ulcerated lesions were all positive at three months post experimental inoculation and negative at six months ( $\mathrm{n}=6$ and 2 respectively). Smears from the liver were positive in $11 / 18(61.11 \%)$ at three months, and in $16 / 18(88.88 \%)$ at six months. Smears from the spleen were $17 / 18(94.44 \%)$ positive at three months and $12 / 18(66.66 \%)$ positive at six months. Isolations and cultures from P. yucatanicus were positive in $61.11 \%(11 / 18)$ and $66.66 \%(12 / 18)$ at three and six months respectively.

\section{DISCUSSION}

This is the first study that examines $P$. yucatanicus clinical and histological features of experimental infection with $L$. (L.) mexicana. As a preliminary study towards the development of a novel experimental model to study LCL caused by $L$. (L.) mexicana, a first and fundamental criterion was to document that both clinical and histological features resembled those described previously in humans with LCL caused by L. (L.) mexicana ${ }^{17}$.
In the present study the most common clinical sign, as reported in patients with $\mathrm{LCL}^{4}$, was a single, ulcerated, rounded small lesion, with elevated borders and necrotic center, observed in $7 / 36(19.44 \%)$ of the $P$. yucatanicus experimentally infected with $1 \times 10^{6} \mathrm{~L}$. (L.) mexicana promastigotes. Moreover, scars developed in P. yucatanicus gathered the criteria for healed lesions of humans with CL, i.e. round or oval shape, smooth surface, depressed, and hypopigmentation ${ }^{37}$.

It must be remarked that absence of local clinical signs was observed in a total of $29 / 36(80.55 \%)$ P. yucatanicus experimentally infected with $1 \times 10^{6} L$. (L.) mexicana promastigotes. As described previously a criterion to incriminate a primary reservoir of Leishmania stated that the infection in a reservoir species should be relatively nonpathogenic, that is, the infection should not lower the survival possibility of the animal since it has to live until the next transmission season, which is more than seven months in the Peninsula. In other words, the immune system of the reservoir must react to the presence of the parasite in such a manner that while preventing irrevocable damage, the parasite evades sterilizing immunity ${ }^{36}$.

There was no difference in the course of the infection linked to $P$. yucatanicus gender. However we do not consider this finding to be conclusive regarding the possible role of sex as demonstrated in the mouse model ${ }^{33}$. More studies will be done regarding the role of sex in susceptibility and/or resistance to the infection by $L$. (L.) mexicana in P. yucatanicus.

The histological picture observed in $P$. yucatanicus experimentally infected with $L$. $(L$. $)$ mexicana was predominantly type III with reference to Magalhaes' classification whereas in patients with CL caused by the same Leishmania species it was type III and IV. Although a possible correlation between clinical and histological features has been examined to understand the immunological response in humans infected with $L$. (L.) mexican $a^{5}$, it has been difficult to establish because such examination depends on a wide range of variables such as the host, the Leishmania species, the time of evolution at the moment of performing the histological study, the inoculation route and the dose of the parasite.

Parasite isolation and culture from the skin of the base of the tail of $P$. yucatanicus was positive in $61.11 \%(11 / 18)$ and $66.66 \%(12 / 18)$ at three and six months, and in smears from the liver and the spleen. With reference to this finding L. major DNA was detected by PCR in the lymph nodes, spleens, bone marrow, and livers of C57BL/6 one year after recovery from infection ${ }^{1}$. Moreover DNA specific for $L$. $(V)$ braziliensis was detected by PCR in scars after clinical cure of $\mathrm{CL}^{24}$. We have reported in a previous study the retention of $L$. (L.) mexicana in 29 naturally infected rodents including six $P$. yucatanicus. These cricetids lived in captivity for up to two years and were tested monthly for the presence of the parasite by cultures of needle aspirates from the base of the tail $^{35}$. These data support the persistence of the parasite in $P$. yucatanicus experimentally infected, as in the human treated or spontaneously healed and in the mouse model of CL.

Since the 1970s parasitic protozoa of the genus Leishmania have provided a useful perspective for immunologists in terms of host defense mechanisms critical for the resolution of infection caused by intracellular pathogens in experimental studies done in the mouse model. The paradigm for the $\mathrm{CD}^{+} \mathrm{T}$-helper 1 (Th1)/Th2 dichotomy is largely based, 
SOSA-BIBIANO, E.I.; VAN WYNSBERGHE, N.R.; CANTO-LARA, S.B. \& ANDRADE-NARVAEZ, F.J. - Preliminary study towards a novel experimental model to study localized cutaneous leishmaniasis caused by Leishmania (Leishmania) mexicana. Rev. Inst. Med. Trop. Sao Paulo, 54(3): 165-9, 2012.

respectively, to the Leishmania major infection in a susceptible/resistant mouse model. Different virulence factors have been identified for distinct Leishmania species, and there are profound differences in the immune mechanisms that mediate susceptibility/resistance to infection and in the pathology associated with disease. These variations not only point to interesting features of the host-pathogen interaction and immunobiology of this genus of parasitic protozoa, but also have important implications for immunotherapy and vaccine development ${ }^{22}$.

A view of leishmaniasis that only considers mouse model infection with $L$. major misses a wealth of interesting immunobiology associated with other species of Leishmania. L. (L.) amazonensis and $L$. (L.) mexicana are associated with quite different patterns of disease following infection of mice when compared with $L$. major. For example, C57BL/6 or $\mathrm{C} 3 \mathrm{H}$ mice, which heal from the L. major infection, develop chronic disease when infected with either $L$. (L.) amazonensis or $L$. (L.) mexicana ${ }^{2}$. Conflicting data may arise in part because different parasite strains or species are being examined, different tissue target (the mice's footpad, the ear, or base of the tail) are being infected, and different doses ("low" $1 \times 10^{2}$ and "high" $1 \times 10^{6}$ ) of metacyclic promastigotes have been inoculated ${ }^{20,27,28}$. In addition immunological studies carried out in humans did not reproduce the Th1/Th2 dichotomy described in the L. major, C57BL/6 and the BALB/c mouse model of $\mathrm{CL}^{18,23,24}$. In summary there is not an experimental model to study LCL caused by $L$. (L.) mexicana.

Although classical laboratory mice have advantages, such as colony maintenance, breed all year round, and a short generation time of 1012 weeks, they have serious limitations when compared with human populations, e.g. they do not exhibit a great variety of natural genetic polymorphisms ${ }^{12}$.

A new approach to study host-parasite relationships bring out because no animal model was available to study the Sin Nombre virus (SNV), a Hantavirus which is a rodent-borne pathogen with a worldwide distribution and that can cause hantavirus cardiopulmonary syndrome (HCPS) in North America. This situation led to the experimental infection of the deer mouse Peromyscus maniculatus, a primary reservoir of the $\mathrm{SNV}^{8}$. However as in the golden hamster experimental model to study visceral leishmaniasis research is hindered by the lack of commercially available immunological and molecular reagents to investigate mechanisms of disease ${ }^{25}$.

In summary, we have found that both clinical and histological features of $P$. yucatanicus infected experimentally with $L$. ( $L$.) mexicana resembled our previous studies done in patients with LCL caused by this parasite. Moreover parasite persistence was recorded as it has been described in patients where CL healed spontaneously after treatment ${ }^{1}$. These results support the utility of $P$. yucatanicus as a novel experimental model to study LCL caused by L. (L.) mexicana.

\section{RESUMEN}

\section{Estudio preliminar sobre un nuevo y original modelo experimental de leishmaniosis cutánea localizada causada por Leishmania (Leishmania) mexicana}

No existe un modelo experimental de la leishmaniosis cutánea localizada (LCL) causada por Leishmania (Leishmania) mexicana. El objetivo del presente estudio fue el de caracterizar los cuadros clínico e histológico de Peromyscus yucatanicus infectados experimentalmente con L. (L.) mexicana. Un total de 54 P. yucatanicus (grupos de 18) fueron inoculados en la base de la cola con $1 \times 10^{6}$ promastigotes de $L$. $(L$. $)$ mexicana. A los 3 y 6 meses posteriores a la infección experimental fueron sacrificados. El grupo control fue inoculado con RPMI-1640. El signo clínico predominante fue una lesión única ulcerada en $27.77 \%(5 / 18)$ y en $11.11 \%(2 / 18)$ P. yucatanicus a los 3 y 6 meses respectivamente. El patrón histológico descrito como inflamación crónica granulomatosa con o sin necrosis se observó en 7/7 (100\%) biopsias de los P. yucatanicus a $\operatorname{los} 3(n=5)$ y $6(n=2)$ meses respectivamente. Los resultados son similares a los cuadros clínico e histológico de la infección por $L$. (L.) mexicana en humanos, y apoyan la posibilidad de utilizar $P$. yucatanicus como un nuevo y original modelo para el estudio de la LCL causada por $L$. $(L$. mexicana.

\section{ACKNOWLEDGMENTS}

We thank the personnel from the animal-house of the Centro de Investigaciones Regionales "Dr. Hideyo Noguchi”, Universidad Autonoma de Yucatán; Dr. Tony Schountz, for editing the manuscript; IQ Silvia Andrade Canto for photography technical assistance.

\section{REFERENCES}

1. Aebischer T, Moody SF, Handman E. Persistence of virulent Leishmania major in murine cutaneous leishmaniasis: a possible hazard for the host. Infect Immun.1993;61:220-6.

2. Aguilar-Torrentera F, Laman JD, Van Meurs M, Adorini L, Muraille E, Carlieri Y. Endogenous Interleukin-12 is critical for controlling the late but not the early stage of Leishmania mexicana infection in C57BL/6 mice. Infect Immun. 2002;70:5075-80.

3. Andrade-Narváez FJ, Simmonds-Diaz EB, Rico-Aguilar S, Andrade-Narváez M, PalomoCetina A, Canto-Lara SB, et al. Incidence of localized cutaneous leishmaniasis (Chiclero's ulcer) in Mexico. Trans R Soc Trop Med Hyg. 1990;84:219-20.

4. Andrade-Narváez FJ, Vargas-Gonzalez A, Canto-Lara SB, Damian-Centeno AG. Clinical picture of cutaneous leishmaniasis due to Leishmania (Leishmania) mexicana in the Yucatan Peninsula, Mexico. Mem Inst Oswaldo Cruz. 2001;96:163-7.

5. Andrade-Narváez FJ, Medina-Peralta S, Vargas-GonzalezA, Canto-Lara SB, Estrada-Parra S. The histopathology of cutaneous leishmaniasis due to Leishmania (Leishmania) mexicana in the Yucatan peninsula, Mexico. Rev Inst Med Trop Sao Paulo. 2005;47:191-4.

6. Beltran F, Bustamante ME. Datos epidemiologicos acerca de la "úlcera de los chicleros" (leishmaniasis americana) en Mexico. Rev Inst Salub Enf Trop. 1942;3:1-28.

7. Biagi F. Sintesis de 70 historias clínicas de leishmaniasis tegumentaria en México (ulcera de los chicleros). Medicina (Mex.). 1953;33:385-96.

8. Botten J, Mirowsky K, Kusewitt D, Bharadwaj M, Yee J, Ricci R, Feddersen RM, Hjelle B. Experimental infection model for Sin Nombre hantavirus in the deer mouse (Peromyscus maniculatus). Proc Natl Acad Sci USA. 2000;97:10578-83.

9. Canto-Lara SB, Cárdenas-Marrufo MF, Vargas-Gonzalez A, Andrade-Narváez FJ. Isoenzyme characterization of Lesihmania isolated from human cases with localized cutaneous lesihmaniasis from the State of Campeche, Yucatan Peninsula, Mexico. Am J Trop Med Hyg. 1998;58:444-7.

10. Canto-Lara SB, Van Wynsberghe NR, Vargas-González A, Ojeda-Farfán FF, AndradeNarváez FJ. Use of monoclonal antibodies for the identification of Leishmania spp. from humans and wild rodents in the State of Campeche, Mexico. Mem Inst Oswaldo Cruz. 1999;94:305-9. 
SOSA-BIBIANO, E.I.; VAN WYNSBERGHE, N.R.; CANTO-LARA, S.B. \& ANDRADE-NARVAEZ, F.J. - Preliminary study towards a novel experimental model to study localized cutaneous leishmaniasis caused by Leishmania (Leishmania) mexicana. Rev. Inst. Med. Trop. Sao Paulo, 54(3): 165-9, 2012.

11. Chable-Santos JB, Van-Wynsberghe NR, Canto-Lara SB, Andrade-Narvaez FJ. Isolation of Leishmania (Leishmania) mexicana from wild rodents and their possible role in the transmission of localized cutaneous leishmaniasis in the State of Campeche, Mexico. Am J Trop Med Hyg. 1995;53:141-5.

12. Guénet JL, Bonhomme F. Wild mice: an ever-increasing contribution to a popular mammalian model. Trends Genet. 2003;19:24-31.

13. Guide for the care and use of laboratory animals. Institute of Laboratory Animal Resources Commission on life Sciences. National Research Council. Washington: National Academy Press; 1996. Available from: http://www.nap.edu/openbook. php?record_id=5140.

14. Itzá-Ortiz MF, Van Wynsberghe NR, Sosa-Bibiano EI, Andrade-Narváez FJ. Reproductive characteristics of a captive colony of big-eared climbing rats (Ototylomys phyllotis). Lab Anim (NY). 2011;40:246-51.

15. Kimblin N, Peters N, Debrabant A, Secundino N, Egen J, Lawyer P, et al. Quantification of the infectious dose of Leishmania major transmitted to the skin by single sand flies. Proc Natl Acad Sci USA. 2008;105:10125-30.

16. Lainson R, Strangsways-Dixon J. Leishmania mexicana: the epidemiology of dermal leishmaniasis in British Honduras. Trans R Soc Trop Med Hyg. 1963;57:242-65.

17. Leader RW, Padgett GA. The genesis and validation of animal models. Am J Pathol. 1980;101(Suppl):S11-S6.

18. Lira R, Doherty M, Modi G, Sacks D. Evolution of lesion formation, parasitic load, immune response, and reservoir potential in C57BL/6 mice following high-and low-dose challenge with Leishmania major. Infect Immun. 2000;68:5176-82.

19. Locksley RM, Pingel S, Lacy D, Wakil A, Bix M, Fowell D. Susceptibility to infectious diseases: Leishmania as a paradigm. J Infect Dis. 1999;179(Suppl 2):S305-8.

20. Louzir H, Melby PC, Ben Salah A, Marrakchi H, Aoun K, Ben Ismail R, et al. Immunologic determinants of disease evolution in localized cutaneous Leishmaniasis due to Leishmania major. J Infect Dis. 1998;177:1687-95.

21. Magalhaes AV, Moraes MAP, Raick AN, Llanos-Cuentas A, Costa JM, Cuba CC. Histopatologia da leishmaniose tegumentar por Leishmania braziliensis braziliensis. 1. Padrões histopatológicos e estudo evolutivo das lesões. Rev Inst Med Trop Sao Paulo. 1986;28:253-62.

22. McMahon-Pratt D, Alexander J. Does the Leishmania major paradigm of pathogenesis and protection hold for New World cutaneos leishmaniases or the visceral disease? Immunol Rev. 2004;201:206-24.

23. Melby PC, Andrade-Narvaez FJ, Darnell BJ, Valencia-Pacheco G, Tryon VV, PalomoCetina A. Increased expression of proinflammatory cytokines in chronic lesions of human cutaneous leishmaniasis. Infect Immun. 1994;62:837.

24. Melby PC, Andrade-Narvaez FJ, Darnell BJ, Valencia-Pacheco G. In situ expression of Interleukin-10 and Interleukin-12 in active human cutaneous leishmaniasis. FEMS Immunol Med Microbiol. 1996;15:101-7.

25. Melby PC, Chandrasekar B, Zhao W, Coe JE. The hamster as a model of human visceral leishmaniasis: progressive disease and impaired generation of nitric oxide in the face of a prominent Th1-like response. J Immunol. 2001;166:1912-20.
26. Mendonça MG, de Brito MEF, Rodrigues EHG, Bandeira V, Jardim ML, Abath FG. Persistence of Leishmania parasites in scars after clinical cure of American cutaneous leishmaniasis: is there a sterile cure? J Infect Dis. 2004;189:1018-23.

27. Menon JN, Bretscher PA. Characterization of the immunological memory state generated in mice susceptible to Leishmania major following exposure to low doses of L. major and resulting in resistence to a normally pathogenic challenge. Eur J Immunol 1996;26:243-9

28. Menon JN, Bretscher PA. Parasite dose determines the $\mathrm{Th}_{1} / \mathrm{Th}_{2}$ nature of the response Leishmania major independently of infection route and strain of host or parasite. Eur J Immunol. 1996;28:4020-8.

29. Norma Oficial Mexicana NOM-062-ZOO-1999. Especificaciones técnicas para la producción, cuidado y uso de los animales de laboratorio. Available from: http:// www.fmvz.unam.mx/fmvz/principal/archivos/062ZOO.PDF

30. Rogers K, DeKrey G, Mbow M, Gillespie RD, Brodskyn CI, Titus RG. Type1 and type 2 responses to Leishmania major. FEMS Microbiol Lett. 2002;209:1-7.

31. Seidelin H. Leishmaniasis and babesiasis in Yucatan. Ann Trop Med Parasitol. 1912:6:295-9.

32. Shattuck GC. Leishmania, Trachoma and foliculosis. In: Shattuck GC. The Peninsula of Yucatan. Medical, biological, meteorological and sociological studies. Washington: Carnegie Institution of Washington; 1933. p. 318-27.

33. Sneider H, Lezama-Davila C, Alexander J, Satoskar AR. Sex hormones and modulation of immunity against leishmaniasis. Neuroimmunomodulation. 2009;16:106-13.

34. Stamper LW, Patrick RL, Fay MP, Lawyer PG, Elnaiem DEA, Secundino N, et al Infection parameters in the sand fly vector that predict transmission of Leishmania major. PLoS Negl Trop Dis. 2011;58:e1288. doi:10.1371/journal.pntd.000128830.

35. Van Wynsberghe NR, Canto-Lara SB, Damián-Centeno AG, Itzá-Ortiz M, AndradeNarváez FJ. Retention of Leishmania (Leishmania) mexicana in naturally infected rodents from the State of Campeche, Mexico. Mem Inst Oswaldo Cruz. 2000;95:595600

36. Van Wynsberghe NR, Canto-Lara SB, Sosa-Bibiano EI, Rivero-Cardenas NA, AndradeNarváez FJ. Comparison of small mammal prevalence of Leishmania (Leishmania) mexicana in five foci of cutaneous leishmaniasis in the state of Campeche, Mexico. Rev Inst Med Trop Sao Paulo. 2009;51:87-94.

37. Wade CM, Kulbokas EJ $3^{\text {rd }}$, Kirby AW, Zody MC, Mullikin JC, Lander ES, et al. The mosaic structure of variation in the laboratory mouse genome. Nature. 2002;420:574

38. Weigle KA, Santrich C, Martinez F, Valderrama L, Saravia NG. Epidemiology of cutaneous leishmaniasis in Colombia: a longitudinal study of the natural history prevalence, an incidence of infection and clinical manifestation. J Infect Dis. 1993;168:699-708.

39. Walton BC. American cutaneous and mucocutaneous leishmaniasis. In: Peters W, KillickKendrick R, editors. The Leishmaniases in Biology and Medicine. Clinical aspects and control. London: Academic Press; 1987. v. 2, p. 642-4.

Received: 6 December 201

Accepted: 16 March 2012 


\section{Revista do Instituto de Medicina Tropical de São Paulo on line.}

Publications from 1987 to the present data are now available on:

http://www.scielo.br/rimtsp

PAST ISSUES 1959-1989 (PDF)

www.imt.usp.br/portal/

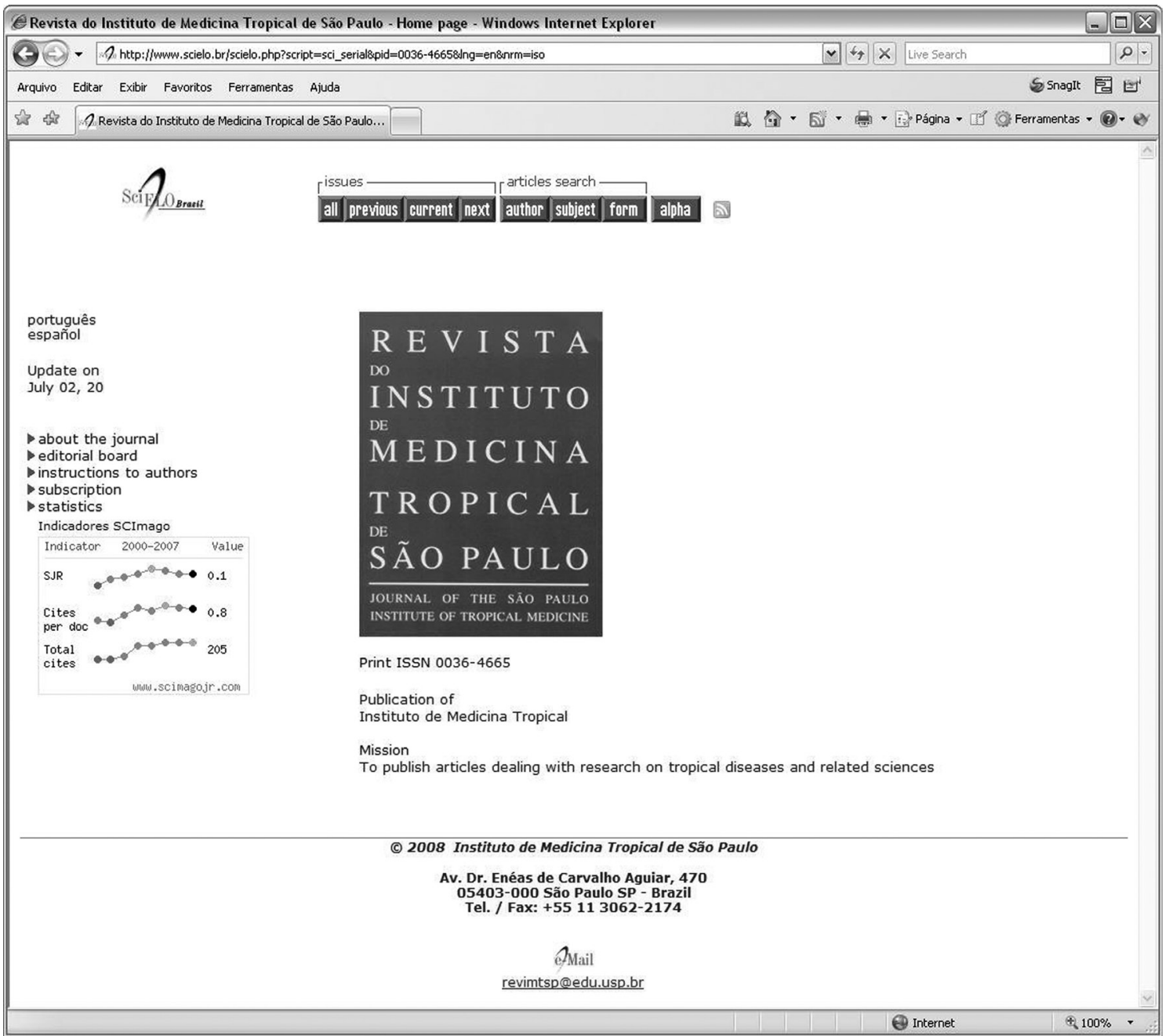

SciELO - The Scientific Electronic Library OnLine - SciELO is an electronic virtual covering a selected collection of Brazilian scientific journals.

The library is an integral part of a project being developed by FAPESP - Fundação de Amparo à Pesquisa do Estado de São Paulo, in partnership with BIREME - the Latin American and Caribbean Center on Health Sciences Information.

SciELO interface provides access to its serials collection via an alphabetic list of titles or a subject index or a search by word of serial titles, publisher names, city of publication and subject.

The interface also provides access to the full text of articles via author index or subject index or a search form on article elements such as author names, words from title, subject and words from full text.

FAPESP/BIREME Project on Scientific Electronic Publications Latin American and Caribbean Center on Health Sciences Information

Rua Botucatu 862 - 04023-901 São Paulo, SP - Brazil

Tel. (011) 5576-9863

scielo@bireme.br 